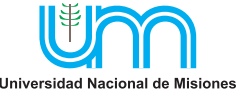

\title{
Modelación y simulación de procesos en la intensificación de instalaciones de la industria química
}

\author{
Cortés Martínez R. ${ }^{a}{ }^{*}$, Ramos Miranda F. ${ }^{a}$, Miño Valdés J.E. ${ }^{b}$, Pérez Navarro O. ${ }^{c}$ \\ González Suárez E. \\ a Facultad de Ingeniería, Universidad de Cienfuegos, Cuba \\ ${ }^{b}$ Facultad de Ingeniería, Universidad Nacional de Misiones, Argentina \\ ' Facultad de Química y Farmacia, Universidad Central "Marta Abreu” de Las Villas, Cuba \\ e-mails: rcortes@ucf.edu.cu, framos@ucf.edu.cu,minio@fio.unam.edu.ar,opnavarro@uclv.edu.cu, \\ erenio@uclv.edu.cu
}

\begin{abstract}
Resumen
El objetivo de este trabajo fue conceptualizar sobre análisis de sistemas incluyendo simulación y modelación matemática buscando representar problemas de la industria química para la intensificación de sus instalaciones. La metodología de trabajo fue la búsqueda bibliográfica. Se describieron las principales características de la modelación matemática y el procedimiento para obtener un modelo similar al comportamiento real del sistema en estudio. Se presentaron análisis, propiedades y clasificación de modelos matemáticos entre otros. La intensificación de procesos en las instalaciones de la industria química fue indispensable para su mejora.
\end{abstract}

Palabras Clave - Industria Química, Intensificación, Modelación, Procesos, Simulación

\begin{abstract}
The objective of this work was to conceptualize on systems analysis including simulation and mathematical modeling seeking to represent problems of the chemical industry for the intensification of its facilities. The work methodology was the bibliographic search. The main characteristics of mathematical modeling and the procedure to obtain a model similar to the real behavior of the system under study were described. Analysis, properties and classification of mathematical models among others were presented. The intensification of processes in the chemical industry facilities was essential for its improvement.
\end{abstract}

Keywords - Chemical Industry, Intensification, Modeling, Processes, Simulation

\section{Introducción}

En la actualidad, la ciencia se está convirtiendo cada vez más en una fuerza productiva directa de la sociedad, lo que hace que las investigaciones aplicadas se encaminen a mejorar y elevar la eficiencia económica de los procesos industriales, la calidad de los productos y la productividad.

Crece incesantemente el empleo de las computadoras para la dirección de los procesos tecnológicos, pues la esencia de esta etapa de la Revolución Científico Técnica es que funciones hasta ahora realizadas por el hombre comienzan a ser obra de las máquinas.

Las ciencias modernas han desarrollado las ideas de la simulación, los métodos de análisis y síntesis de los sistemas, por lo que la modelación matemática, interpretada, como la representación de la verdad relativa de un lado del fenómeno, pertenece al campo de las ciencias, que estudia la 
cibernética matemática y es un fruto del desarrollo científico moderno, dado que las ideas de cada época se ven en sus técnicas. [1]

La ciencia y la introducción de los resultados científicos en la producción, adquieren relevantes funciones sociales por lo que deberán estar orientadas al desarrollo armónico y proporcional de la ciencia de manera que contribuya al desarrollo integral de la técnica, la producción y la economía [2].

El objetivo de este trabajo fue conceptualizar el análisis de sistemas incluyendo simulación y modelación matemática para representar los problemas de la industria química buscando intensificar sus instalaciones.

\section{Materiales y métodos.}

El método de investigación aplicado fue la revisión sistemática de literatura sobre el tema. En el acopio de la documentación requerida se utilizó el motor de búsqueda Google, con el cual fue posible registrar publicaciones seriadas, resultados de investigación y libros. La revisión y análisis del acervo documental obtenido se interpretó cualitativamente.

\section{Resultados y discusión}

\section{Teoría de los Sistemas y Modelación Matemática}

La revolución científico técnica moderna da inicio a la era de la revolución automatizada, de manera que las ciencias se transforman en una fuerza productiva directa.

La complejidad del desarrollo tecnológico exige que para abordar las tareas técnicas contemporáneas, se considere que la ciencia se adelanta a la técnica y a la producción en su desarrollo. Se plantea cada vez de forma más aguda los problemas vinculados a la síntesis de los conocimientos, a la elaboración lógica de la información, siendo de carácter integral el rasgo específico del desarrollo de las ciencias modernas, lo que es expresión de la dialéctica del objeto mismo, dando vida a los diversos métodos de síntesis de los conocimientos relativos a diferentes ciencias. A la par y por ello en las ciencias modernas el estudio de los sistemas poseedores de propiedades comunes, se ha difundido ampliamente, planteando el problema de la elaboración de la metodología de las investigaciones sistemáticas. Esta necesidad del desarrollo ha sido satisfecha con los métodos matemáticos, que con la simulación de los fenómenos se interese por una de las cualidades del pensamiento humano: la previsión y el pronóstico; por lo que los métodos matemáticos imprimen un carácter cualitativamente distinto al progreso técnico.

Con el desarrollo de la cibernética fueron ampliándose sus aplicaciones, y se concrete una definición general más apropiada como la dada por Klaus (1974) como "la teoría de las relaciones de posibles sistemas dinámicos auto regulados con las partes que lo componen" o una más general y menos comprometedora sería: "la cibernética es la ciencia de los sistemas cibernéticos". [3]

El método científico de investigación aplicable a todas las esferas de las ciencias y la técnica, es la teoría de los sistemas, pues la teoría de los sistemas es un método general de investigación científica que transforma los distintos principios de la metodología de investigación 
de tal forma que estos adquieren significado heurístico en el conocimiento científico especializado. [4]

La teoría de los sistemas surge como una necesidad de la matemática al estudiar los sistemas Así, esta comprende la teoría que se ocupa de las relaciones entre la estructura y la función del sistema y las interacciones entre las partes y el todo del sistema. Para Kafarov (1976), la matemática de los procesos de la industria química se ocupa de las relaciones entre los equipos de los procesos continuos (relaciones entre elementos del sistema), así como las uniones entre los equipos y sus partes y además, del comportamiento del sistema dependiendo de la influencia del ambiente (relaciones entre estructura y función). [5]

En la teoría de los sistemas, el análisis de los mismos es la metódica para la obtención de los resultados en el estudio activo, es decir, la acción y dirección de un sistema, para ello se requieren, métodos de modelación que sean capaces de representar los aspectos y rasgos que se desean estudiar en el comportamiento de los sistemas.

La Teoría de los Sistemas, que junto con la teoría de la información, la teoría de la regulación, la teoría de los juegos y la teoría de los algoritmos, conforman la cibernética, tiene como concepto central el de "sistema", aunque una definición general de sistema se hace difícil; podemos referirnos a la de Kafarov (1976): La totalidad de los procesos químicos físicos que se desarrollan, así como los medios técnicos que lo posibilitan. [5]

Desde el punto de vista de la cibernética son los sistemas dinámicos los que se estudian como parte de ella. Las propiedades generales que estos poseen son las siguientes:

1) Integridad, 2) Complicación. 3) Estabilidad dinámica multidimensional. 4) Controlabilidad. 5) Transición en el tiempo. 6) Procesamiento y almacenamiento de la información, 7) Estructura jerárquica. 8) Independencia relativa de las partes del sistema, 9) Diferenciabilidad cualitativa de los elementos.

\section{Clasificación de los Sistemas.}

La clasificación de los modelos matemáticos dinámicos complejos puede efectuarse desde diferentes principios. Seguiremos el siguiente: [6]

Sistemas Cerrados: aquellos en que existen solamente elementos internos. Las salidas de sus elementos son al mismo tiempo entradas de otros.

Sistemas Abiertos: son aquellos en los cuales por lo menos un elemento no tiene entrada o salida a otro elemento.

Sistemas lineales: entre las salidas y las entradas del sistema existen relaciones lineales.

Sistemas no lineales: no existen relaciones lineales entre las salidas y las entradas del sistema, estos sistemas bajo determinadas consideraciones pueden llegar a linealizarse.

Sistemas determinísticos: son aquellos en los cuales las variables que los describen cambian de acuerdo con regularidades fijadas exactamente, así como que sus valores estén fijos determinan claramente el estado del sistema.

Sistemas estadísticos: las variables del sistema son variables casuales, cuya distribución es desconocida pero que pueden conseguir por medio de la información de muestras. 
Sistemas estocásticos: Son aquellos sistemas estadísticos donde las variables que lo escriben dependen de otro parámetro $\mathrm{t}$ (en general el tiempo), o sea que está en presencia de variables casuales con variación con respecto al tiempo.

Sistemas discretos: aquellos en los cuales cada variable del sistema puede tomar una serie de valores $\mathrm{Ci}$; $\mathrm{i}$ : 1, 2, 3..., y, para ello los $\mathrm{Ci}$ permanecen aislados, o sea que para cada $\mathrm{Ci}$ existe un ambiente, que no puede existir otro $\mathrm{Cj} ; \mathrm{J}=\mathrm{i}$ de dicha variable $\mathrm{C}$.

Sistemas Continuos: son aquellos en los cuales cada variable del sistema puede tomar todos los valores posibles en un intervalo determinado. De acuerdo a su carácter se pueden diferenciar sub sistemas cualitativos del sistema global como sigue: a) Sistemas de masas, b) Sistemas de energía. d) Sistemas de información.

Partiendo de las definiciones anteriormente dadas, puede concluirse con las siguientes ideas básicas sobre los sistemas:

- Se crea con un objetivo preciso. Este objetivo condiciona al sistema, así como a su actividad y a su conformación interna, por tanto a su complejidad.

- Los sistemas de procesos tienen la función de realizar transformaciones industriales de masa y energía.

- Los elementos que componen el sistema de procesos se agrupan, en procesos de transformación de energía y masa para el transporte y almacenamiento de ellas.

- Las relaciones se componen de las corrientes de masa y energía que crean los acoples entre los elementos del sistema.

- Los sistemas cibernéticos poseen características homeostáticas, es decir, son capaces de conservar la estabilidad de sus parámetros fundamentales pese a los cambios del medio

- Los sistemas pueden ser descrito por un conjunto de variables que reflejan determinadas propiedades de los elementos del mismo

- Entre el sistema y el medio y a la vez entre los diferentes subsistemas existen canales a través de los cuales se transmite información

- La organización o acoplamiento de los subsistemas de un sistema se realiza a partir de tres tipos de flujos fundamentales: secuenciales, en paralelo y retroalimentación.

Los planos de consideración de los sistemas de procesos son: [2]
a) Grupos de unidades,
b) Etapas de Procesos,
c) Procesos completos,
d) Fábricas de transformación de materiales,
e) Sistemas combinados de transformación de materiales,

Por otro lado, los elementos de un sistema pueden clasificarse de acuerdo a:

1. Número de unidades de entrada y salida,

2. De acuerdo con el comportamiento en el tiempo.

3. Según las variables de decisión, e) Según la función técnica del proceso 
Los sistemas de procesos pueden clasificarse en dos grupos:

1. Según la función en:

a) Sistema para la transformación de materiales,

b) Sistemas para el suministro de materiales auxiliares,

c) Sistemas para el suministro de energía,

d) Sistemas de información

2. Según la estructura en:

a) Acople en serie,

b) Acople en cascada,

c) Acople en paralelo,

d) Acople de desviación,

e) Acople de recirculación

\section{Análisis de Sistemas.}

El Análisis de Sistemas trata básicamente de determinar los objetivos y límites del sistema objeto de análisis, caracterizar su estructura y funcionamiento, marcar las directrices que permitan alcanzar los objetivos propuestos y evaluar sus consecuencias. Dependiendo de los objetivos del análisis se pueden encontrar dos problemáticas distintas:

- Análisis de un sistema existente para comprender, mejorar, ajustar y predecir su comportamiento.

- Análisis como paso previo al diseño de un nuevo sistema-producto.

El Análisis de Procesos Tecnológicos se puede realizar bajo un aspecto limitado, unilateral del proceso, o con un enfoque multilateral más complejo.

Los Análisis Parciales, aunque son también necesarios para la economía de las empresas, sólo brindan soluciones parciales, que no necesariamente tienen que coincidir con las que se logran con un enfoque multilateral más complejo, que por otro lado requiere de la participación de personal calificado de varias ramas de la ciencia que laboren de forma coordinada en la búsqueda de las reservas del proceso estudiado. [7]

En la literatura internacional se reportan numerosas referencias a diferentes análisis parciales, un amplio campo de estos trabajos lo ocupan, los análisis de las condiciones tecnológicas en los procesos de la industria química concentrándose en la influencia de la conducción y la selección del parámetros del proceso sobre el incremento de las capacidades de producción; sobre la fiabilidad de los procesos; sobre los consumos específicos de producción y los balances de materiales y energía. Los incrementos de las capacidades de producción deben sustentarse a programas de intensificación o llámese de mejoras y que depende de la particularidad de cada industria.

En cualquier caso, se pueden agrupar formalmente las tareas que constituyen el análisis en una serie de etapas que se suceden de forma iterativa hasta validar el proceso completo:

Conceptualización: Obtener una visión de muy alto nivel del sistema, identificando sus elementos básicos y las relaciones de éstos entre sí y con el entorno. 
Análisis funcional: Describe acciones y transformaciones que tienen lugar en el sistema, las cuales se describen como funciones que reciben entradas y producen salidas.

Análisis de condiciones (o constricciones): Debe reflejar todas las limitaciones impuestas al sistema que restringen el margen de las soluciones posibles. Estas se derivan a veces de los propios objetivos del sistema:

- Operativas: restricciones físicas, ambientales, de mantenimiento, de personal, etc.

- De calidad: fiabilidad, mantenibilidad, seguridad, convivencialidad, etc.

En ciertos casos se presentan limitaciones en los recursos utilizables:

- Económicos, reflejados en un presupuesto.

- Temporales, que suponen unos plazos a cumplir.

- Humanos.

- Metodológicos, que conllevan la utilización de técnicas determinadas.

- Materiales, como espacio, herramientas disponibles, etc.

Construcción de modelos: Una forma habitual y conveniente de analizar un sistema consiste en construir un prototipo (un modelo material o conceptual) del mismo.

Validación del análisis: A fin de comprobar que el análisis efectuado es correcto y evitar la posible propagación de errores, en la fase de diseño es imprescindible proceder a la validación del mismo. Para ello se verifica que:

- El análisis debe ser consistente y completo.

- Si el análisis se plantea como un paso previo para realizar un diseño, habrá que comprobar además que los objetivos propuestos son correctos y realizables.

\section{Modelación matemática}

Un modelo matemático se define, de manera general, como una formulación o una ecuación que expresa las características esenciales de un sistema físico o de un proceso en términos matemáticos. [8]

Los modelos tienen dos propósitos básicos, con formas fundamentales y de acuerdo con estos propósitos pueden ser:

a. Modelos dinámicos, tipo de ecuaciones generalmente diferenciales, para los estudios de control automático y afines.

b. Modelos estáticos, tipos de ecuaciones generalmente algebraicas, para estudios de diseño técnico y optimización técnico económica.

La forma y uso de los modelos también determinan indirectamente los límites de su tamaño físico en términos de número de variables que se estimen y la cantidad de ecuaciones implicadas, así como el tamaño y la complejidad de los medios físicos que se consideren.

La descripción cuantitativa de los sistemas de procesos, es una de las tareas de interés en la actualidad, para lograr este objetivo, una de las condiciones previas, es que la cantidad de información disponible permita describir, las relaciones entre los elementos y dentro de los elementos, de manera que con el modelo obtenido de un sistema real, se pueda en determinada etapa del proceso del conocimiento sustituir al sistema real, y por medio de su investigación y 
estudio obtener la información deseada sobre el sistema real, por ello, la calidad de un modelo matemático está determinada por la precisión con que coincidan los procesos en el sistema real con los procesos obtenidos mediante el modelo, y debe existir:

1. Concordancia objetiva con el propio sistema;

2. Posibilidad de sustituir el sistema en el proceso del conocimiento;

3. Propiedad de suministrar información práctica sobre el sistema;

4. Determinadas reglas mediante las cuales la información del modelo pueda transformarse en información del sistema.

\section{Propiedades de los modelos}

El estudio de los modelos como método fundamental del conocimiento debe servir para conocer las correlaciones cuantitativas propias de los fenómenos y con ello mejorar el sistema para direccionar él o los procesos como caso de estudio. Entonces la modelación es un método de manejo práctico o teórico de un sistema por medio del cual debe ser estudiado el sistema, pero no como tal sino por medio de un procedimiento auxiliar natural o artificial, el cual desde el punto de vista de los intereses planteados concuerda con el sistema real que se estudia. Dicho sistema en determinadas etapas del proceso del conocimiento, ser capaz de sustituir al real y por medio de su investigación y estudio, suministrar la información deseada sobre el sistema real.

Entre las propiedades generales de los modelos se encuentran:

1. Los modelos son representaciones conscientemente desarrolladas y simplificadas de una circunstancia. La construcción de modelos es una forma particular de la capacidad de abstracción del hombre.

2. Los modelos contienen las facetas esenciales y decisivas del proceso en dependencia del fin del estudio.

3. Los modelos en general brindan nuevos conocimientos.

4. Los modelos se comprueban mediante la comparación de sus salidas con los resultados de los procesos reales.

5. Los modelos son sistemas de sustitución ya que sustituyen un original complejo para aclarar el objeto de investigación.

En resumen, puede plantearse que las tres propiedades fundamentales de los modelos son la habilidad de abstracción como operación mental para separar las propiedades de un objeto, habilidades de semejanza como conjunto de características comunes para establecer su naturaleza y habilidades de extrapolación de los resultados como aplicación de un criterio lógico conocido de otros casos con el fin de extraer conclusiones o hipótesis a futuro.

\section{Clasificación de los modelos}

Los modelos pueden clasificarse atendiendo a muchos factores, entre otras clasificaciones tenemos las siguientes:

1. De acuerdo a la teoría o técnica utilizada en su construcción, se clasifican en tres tipos de modelos y sus combinaciones: 
a. Modelos de fenómenos de transporte. Basados en principios físico químicos

b. Modelos de Balances de Población Aplicando los principios de Balances de Población.

c. Modelos Empíricos: Su construcción se basa en técnicas de ajuste de datos empíricos.

2. Desde el punto de vista de la naturaleza de las ecuaciones, se clasifican en pares opuestos como sigue:

a. Modelos determinísticos o estocásticos.

b. Modelos estacionarios o dinámicos.

c. Modelos de parámetros combinados o de parámetros distribuidos.

Otras clasificaciones de los modelos son dadas en la literatura, según los aspectos de los objetos que el modelo refleja, a saber, modelos de estructuras, funcionales, de comportamiento, combinados. [9]

\section{Confección, formulación y aplicación de modelos en la industria química y fermentativa}

El planteamiento de un modelo matemático de un proceso exige, por un lado el entendimiento del desarrollo del proceso y por otro, que la persona encargada del desarrollo del modelo posea capacidad de abstracción que le permita integrar por medio de formulación matemática todas las peculiaridades del objeto, debe además ser capaz de despreciar los factores no influyentes y de simplificar el modelo en todo lo que sea posible. [10]

Los modelos matemáticos de los equipos de un proceso, en conjunto dan forma al modelo matemático. Un modelo es la representación matemática o grafica de la realidad que es utilizada para plantear un problema, normalmente de manera simplificada en términos relativos y planteados desde un punto de vista matemático, aunque también puede tratarse de un modelo físico. [11]

Para llegar a establecer un modelo global, es necesarios iniciar con modelos iniciales y que deben ser mejorados cada vez, para alcanzar un cierto grado de éxito de un proceso e particular. El trabajado experimental eficiente finaliza en un modelo matemático, apoyado en el método científico, que para establecerlo los investigadores deben considerar la guía que se representa en la figura 1 .

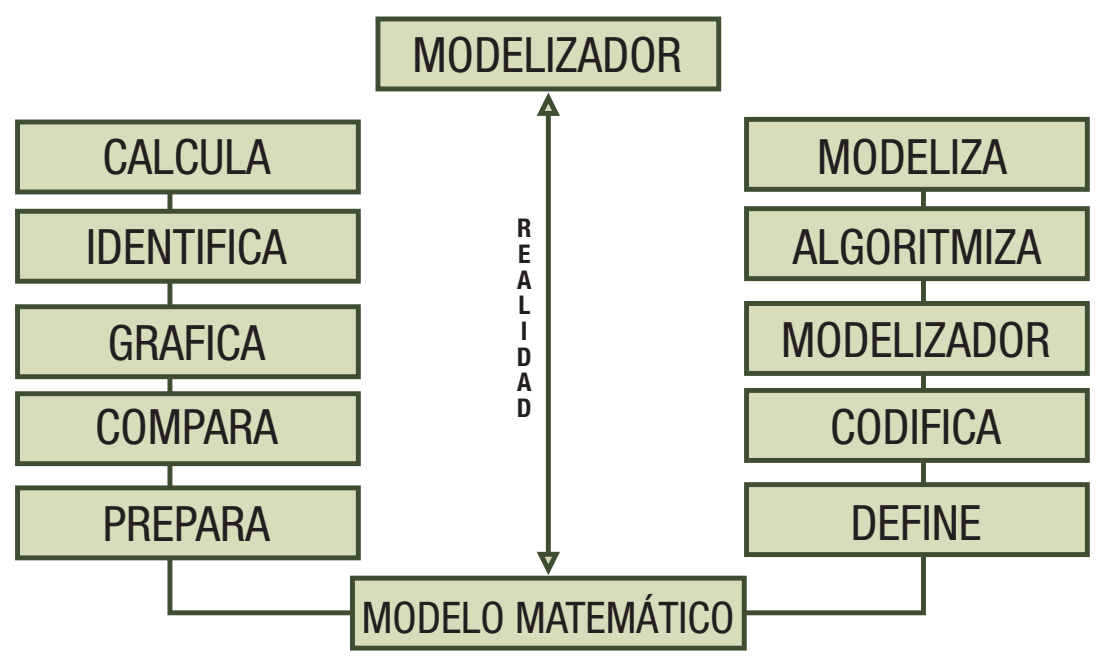

Fig. 1. Procedimiento a seguir para definir un modelo matemático. 
Esta tarea, por su complejidad y requerimientos hace que las exigencias en el modelo y su planteamiento sean elevados. Los programas heurísticos (deductivos) juegan un papel fundamental en el desarrollo de una tarea de tal magnitud, ya que el fin de la confección de dichos programas es el obtener experiencias metódicas para la estructuración efectiva del trabajo creador del hombre.

Si analizamos este aspecto encontramos en la confección de un modelo matemático un movimiento de la contemplación, al pensamiento abstracto y de ahí a lo concreto pensado (que no es otra cosa que el modelo), como reflejo ideal del mundo real, lo que al comparar su adecuación completa se conoce como contemplación viva. La modelación como parte de la Teoría de los Sistemas, está relacionada con una serie de principios que son la base de los estudios posteriores de la modelación. [12]

\section{Etapas Generales para el Desarrollo de modelos}

Los pasos generales que llevan a un modelo matemático global han sido relacionados por Kafarov (1976) y una representación gráfica es la Figura 2.

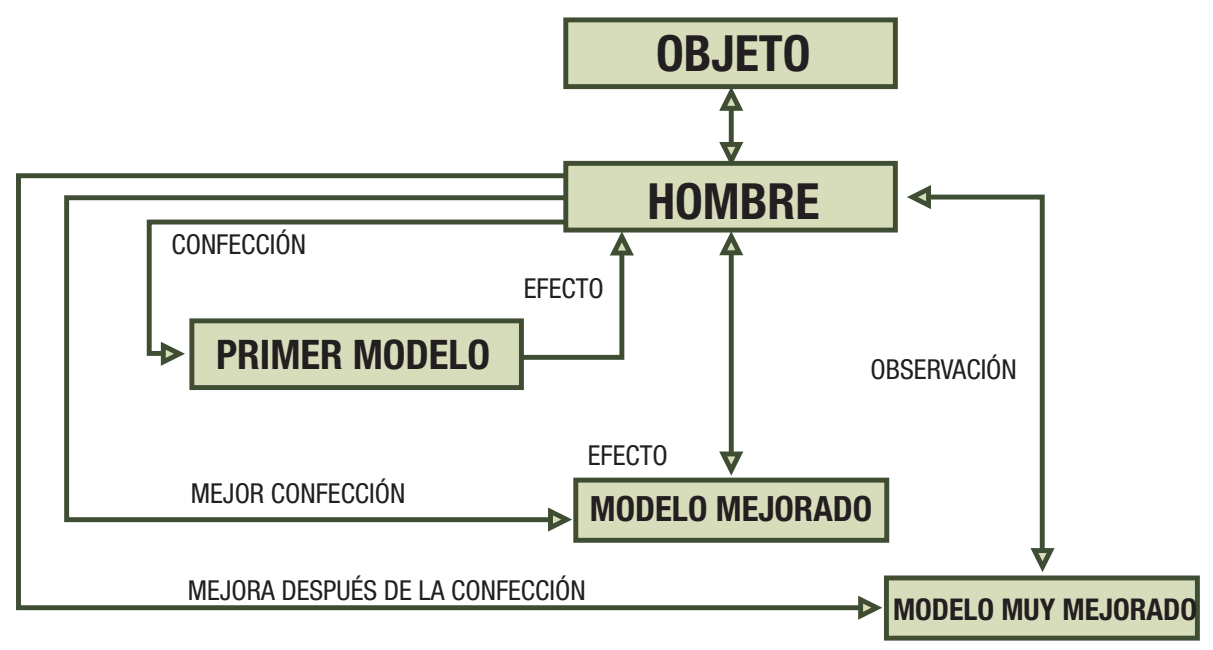

Fig. 2. Representación gráfica para la mejora en la elaboración de un modelo. Fuente: [5]

La formulación de modelos debe ser un proceso progresivo, donde inicialmente se formule un modelo de poca elaboración y luego se refine gradualmente hasta obtener el equilibrio mencionado anteriormente. Generalmente, es un error tratar de formular en el inicio del proceso el modelo más complejo y elaborado.

La Modelación Matemática se lleva a cabo cumpliendo los siguientes pasos: [13]

a) Identificación del problema o proceso a resolver, junto con los objetivos, que deben ser claros y concisos, con miras a obtener un resultado.

b) Conocimiento de los aspectos cognitivos que rigen el problema, por medio de leyes, teorías y conceptos inmersos en la situación objeto de trabajo.

c) Formulación de la situación-problema en términos matemáticos (matematización), mediante ecuaciones y/o relaciones matemáticas, identificando para ello las variables, parámetros y los supuestos a que haya lugar. 
d) Solución del problema y/o proceso matemático obtenido. Para ello se hará uso de herramientas tales como tablas, gráficas, ecuaciones diferenciales, estadística, investigación de operaciones.

e) Comparación del modelo con la situación real. En función de esto, es necesario analizar los resultados obtenidos, verificando si las respuestas alcanzadas son correctas, adecuadas o no, y además si se generan soluciones extrañas.

f) Análisis de las restricciones al modelo. Es importante tener en cuenta las suposiciones hechas, así como el marco donde se va a limitar el dominio de la solución obtenida.

g) Escenario de aplicación del modelo, así como su interpretación. Es aquí donde se evidencia el cumplimiento de metas, teniendo en cuenta sus restricciones, si las hubiere.

La estrategia general para la elaboración de modelos sigue una trayectoria definida y razonable, la que aunque no debe ser aplicada mecánicamente pues las peculiaridades de cada sistema dan lugar a divergencias en el desarrollo de los métodos, se resume en los siguientes pasos lógicos: [5]

\section{Estudio preliminar:}

Todo trabajo de modelación tiene como premisa un estudio preliminar en el cual se definan los problemas fundamentales para el planteamiento del modelo. El primer aspecto es reflejar los objetivos que se persiguen con el modelo, es decir los fines específicos del modelo. Para la optimización deben decidirse los criterios deseados como parámetros de optimización y además conocerse las relaciones fenomenológicas del proceso.

Es también necesario conocer sus limitaciones espaciales, constructivas y de procedimiento. Las limitaciones espaciales las fijan las instalaciones o equipos en los cuales el proceso se desarrolla y también las relaciones con los alrededores. Los constructivos están determinados por aspectos tales como: tipo de construcción, elementos de construcción, dimensiones de los equipos etc. En este paso, se incluye el análisis crítico de la literatura y de los modelos existentes para procesos análogos y debe terminar con una corroboración de los objetivos propuestos y un planteamiento de los modelos que pueden ser empleados en la solución de la tarea.

\section{Elaboración del modelo matemático detallado:}

El modelo matemático de un proceso tecnológico debe describir en forma de relaciones lógicas y/o matemáticas la totalidad de los procesos físicos químicos que ocurren en el sistema analizado, para ello el estudio preliminar sirve como base y se podrán plantear las consideraciones de los procesos elementales esbozados en éste.

En el análisis de estos procesos elementales se combina las consideraciones físicas y físico químicas con Descripciones del tipo "Caja Negra".

En la elaboración de los modelos se comienza por los elementos y para ello pueden aprovecharse los modelos que en el estudio preliminar se hayan seleccionado o elaborado, después se acomete el estudio de la modelación del proceso completo. El modelo del proceso global se logra a través de la integración lógica y articulada de los modelos de los elementos y hasta ese momento tendrá un carácter teórico ya que los parámetros en incluidos no han sido 
evaluados. Los algoritmos y formulaciones elaboradas en esta etapa, serán sometidos en las etapas posteriores a una necesaria comprobación que puede tener como resultado que este paso tenga que repetirse.

\section{Fijación de los valores de los parámetros empíricos: [14]}

En la modelación matemática de los procesos tecnológicos de la industria química es problemático la fijación de los parámetros empíricos debido a la complejidad de los procesos y los cambios en las corrientes másicas. Por otro lado en muchos casos los valores obtenidos a nivel de laboratorio y planta piloto no son extrapolables a escala industrial o solo lo son en un rango limitado.

La obtención de estos parámetros empíricos puede hacerse partiendo de valores establecidos, o determinándose a través de experimentos en equipos pilotos adecuados, o lo que es mucho mejor en la misma fábrica en dependencia de las posibilidades reales.

Los diseños de experimentos y los métodos estadísticos de procesamiento de la información experimental y de los datos del control operacional industrial son un valioso apoyo en la determinación de los parámetros empíricos. Es importante además, la selección de los instrumentos de medida, su exactitud y error relativo. Confección y puesta a punto de los programa para la computadora.

Para el desarrollo de modelos matemáticos, se hace necesaria la utilización de computadoras como herramienta para la realización de los cálculos. Esto se logra por medio de un sistema de programas, que de una forma coordinada lleva a vías de hecho la tarea planteada.

\section{Desarrollo de los cálculos numéricos con el modelo:}

En esta etapa, el modelo pasa a ser el objetivo de estudio por medio del cual se investiga el comportamiento del proceso real, sin necesidad de realizar costosas investigaciones obviando también la influencia casual de determinadas variables. Para ello es un requerimiento que el modelo empleado sea adecuado para la descripción del proceso tecnológico en los límites previamente establecidos. Comprobación de la adecuación del modelo matemático y adaptación al proceso real.

La verificación de la adecuación del modelo permite determinar la concordancia de los resultados de este con los reales del proceso.

Para ello se comparan los resultados experimentales del proceso con aquellos obtenidos por el modelo partiendo de los mismos datos de entrada. Esto permite determinar el grado de concordancia de los resultados del modelo con los reales y si el modelo es capaz de reflejar adecuadamente el proceso para su estudio.

Este procedimiento para resolver problemas ingenieriles a partir de modelos matemáticos se resumen en la Figura 3. 


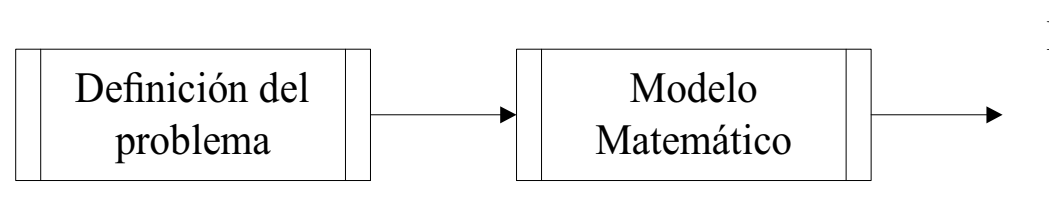

Herramientas para resolver problemas: computadoras, estadísticas, métodos numéricos, gráficas, etc.

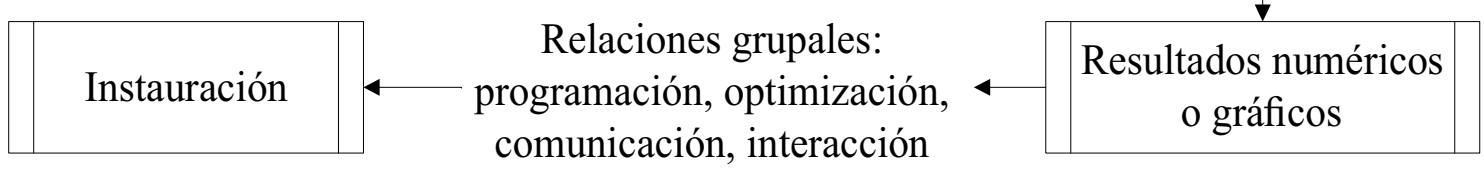
publica, etc.

Fig. 3. Proceso de solución de problemas de ingeniería. Fuente [8]

\section{Optimización de los modelos a través de la Simulación}

La obtención de modelos fiables puede requerir del uso de algoritmos de optimización que permitan conocer los parámetros del proceso a partir del modelo propuesto y los datos experimentales que se manejan. En la Figura 4 se puede observar el procedimiento para optimizar los modelos matemáticos. [15]

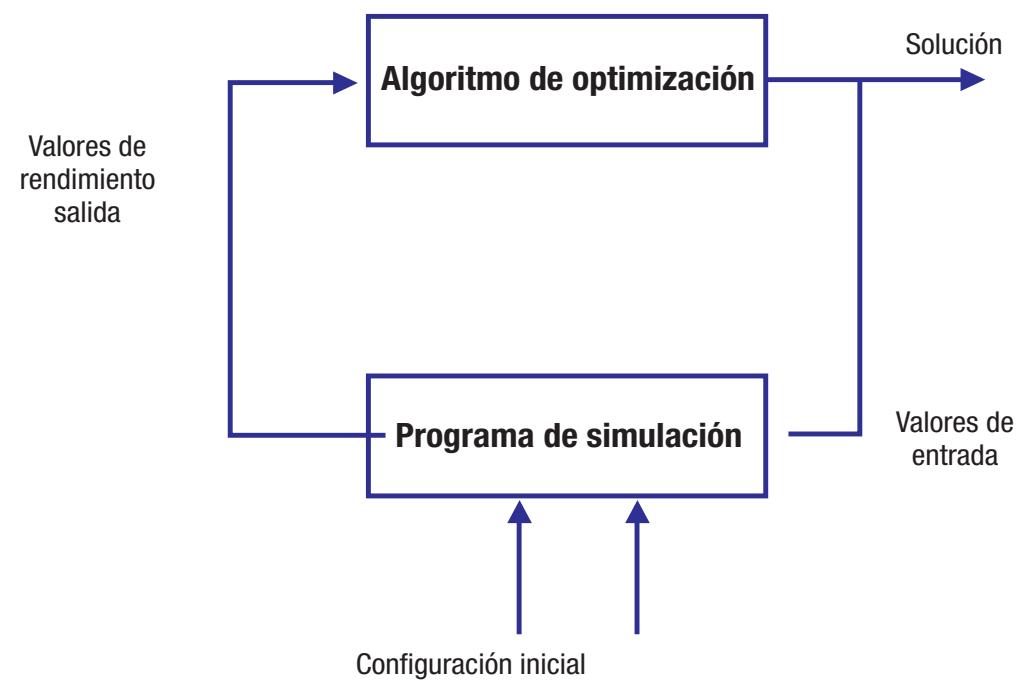

Fig. 4. Dinámica de optimización de modelos.

En la actualidad la modelación y simulación que desarrollan los ingenieros tienen un soporte teórico imprescindible pero su implementación y ejecución práctica se realiza usando aplicaciones informáticas. Es una actividad indispensable cuando nos enfrentamos con el análisis y diseño de sistemas multidisciplinares de cierta complejidad. El objetivo es ayudar o dar el soporte necesario al diseñador durante el proceso de diseño, análisis y diagnosis de sistemas ingenieriles. El software debe complementar el talento del diseñador para que este pueda modelar y simular de forma lo más eficientemente posible. El software hace posible establecer una valoración final antes de que los sistemas sean construidos, y pueden aliviar la necesidad de experimentos caros y dar soporte a todas 
las etapas de un proyecto desde el diseño conceptual, pasando por el montaje hasta llegar a su funcionamiento.

Se puede establecer que estas aplicaciones informáticas pueden clasificarse en los siguientes tipos: [6]

- Matemáticas: Son aquella donde se definen modelos matemáticos y se resuelven problemas de esta ciencia (por ejemplo: resolver ecuaciones y sistemas de ecuaciones, cálculo de derivadas e integrales, resolver ecuaciones diferenciales, etc.) dentro de los que podemos mencionar con características generales el CurvExpert y el Derive

- Ingenieriles: Estas aplicaciones resuelven problemas ingenieriles usando métodos matemáticos. Su característica más notoria es que los datos de entrada y las soluciones son los del problema ingenieril. Generalmente el procesamiento matemático está dado en segundo plano y es transparente para el usuario de la aplicación. Dentro de la industria química podemos mencionar como los más usados el paquete de AspenPlus y Hysys.

- Mixtas: Se trata de aplicaciones donde se presentan herramientas para la modelación y simulación usando directamente los conceptos matemáticos pero también se presentan modelaciones y simuladores que trabajan en el lenguaje y con conceptos ingenieriles. Un ejemplo de estas aplicaciones es el MatLab.

\section{Intensificación de procesos}

Para lograr la intensificación de los procesos tecnológicos de la industria química se utiliza el Análisis de Procesos, el cual consiste en un amplio análisis científico-técnico y técnicoeconómico de un proceso existente o concebido, en lo referente a las posibilidades de realización óptima de los objetivos previstos, por ello constituye un elemento importante para tomar decisiones más científicas y responsables.

El Análisis de Procesos sirve para descubrir las partes débiles en el proceso de producción correspondientes y para la creación de medidas para su eliminación parcial o completa lo que conduce a un aumento de su efectividad permitiendo de esta forma un mejor aprovechamiento de las materias primas, la energía y los medios de trabajo así como un aumento de los grados de eficiencia de la fuerza de trabajo y el mejoramiento de las condiciones materiales bajo las cuales ellos laboran. La intensificación de la producción mediante el Análisis Complejo de Procesos, requiere la elaboración de objetivos económicos en cada industria de forma concreta, entre lo que se encuentra:

- Aumento de la calidad y la cantidad de los productos elaborados.

- Disminución de los consumos específicos y absolutos de materiales y energía.

- Mejoramiento de las condiciones de trabajo.

- Ahorro de fuerza de trabajo.

- Disminución de la contaminación ambiental

Por consiguiente, la función clave de la intensificación de procesos es su optimización, y como ya se ha visto anteriormente la optimización de procesos se hace con apoyo de modelos 
matemáticos, elaborados a partir de los valores reales del comportamiento de los propios procesos [16] y la optimización de estos modelos se hace, en la mayoría de los casos debido a la complejidad de estos, mediante la simulación.

Los modelos deben siempre considerarse como un soporte o ayuda para la toma de decisiones y nunca como el reemplazo de las personas que toman las decisiones. Si se formula un modelo pensando que sus resultados van a resolver todos los problemas de decisión y van a ser implementados "a ojos cerrados," se cae en un grave error que puede traer consecuencias impredecibles. Existen en la realidad muchos factores que no pueden ser involucrados en modelos matemáticos y que sólo los grupos de personas que toman las decisiones pueden evaluar. Por lo tanto, desde este punto de vista, los modelos son una poderosa herramienta para este proceso, pero no el fin mismo de la decisión.

\section{Conclusiones}

Uno de los aspectos básicos de la modelación matemática es el de considerar el suficiente grado de abstracción de la realidad, de tal forma que el modelo tenga el suficiente realismo, contra la posibilidad de que sea resuelto en un tiempo de simulación aceptable. El arte de modelar consiste precisamente en identificar las características fundamentales del sistema a ser involucradas en el modelo y en diseñar modelos que permitan ser resueltos en forma eficiente. Desde este punto de vista es tan importante y fundamental formular un modelo, como encontrar sus soluciones en un tiempo computacional aceptable, para después analizarlas e implementar las que se consideren mejores.

En la actualidad la intensificación de procesos está fuertemente ligada a la modelación matemática y la simulación pues el avance en este campo de la ciencia permite una mejor toma de decisiones y así un aumento en la calidad de las industrias químicas.

\section{Referencias}

[1] González, E (1991). Aplicación del Análisis de Procesos en intensificación de distintas industrias de Cuba. Tesis de disertación de Doctor en Ciencias. Universidad Central de Las Villas. Cuba.

[2] González Suárez, E. W. F. Quezada Moreno, I. Y, González Herrera. D. N. Concepción Toledo, Miño Valdés J.E. (2018). "Gestión del conocimiento para la industria química y fermentativa con apoyo de la optimización" EdUNaM. ISBN: 978-950-766-133-4, Posadas 2018 González et al, 2018

[3] Klaus, G. (1974). Systrems Informationes Strategien. V.E. H. verlag Technik, Berlin.

[4] Orudzhev, Z. M. (1974) La dialéctica como sistema. Editorial de Ciencias Sociales, Ciudad de La Habana.

[5] Kafarov, V. (1976). Cybernetic Methods in Chemistry and Chemical Engineering. MIR, Moscow, pp. 464-470. Moscú

[6] Legrá-Lobaina, A. A. (2011). Modelación Matemática y Simulación de Sistemas de Ingeniería. Monografía. Departamento de Matemáticas. Instituto Superior Minero Metalúrgico de Moa 
[7] Himmelblau, D.M. (1970). Process Analysis by Statistical Methods. New York, John Wiley \& Sons. EEUU.

[8] Chapra, S. C., \& Canale, R. P. (2011). Capítulo 1: Modelos matemáticos y solución de problemas de ingeniería. En Métodos numéricos para ingenieros. Sexta edición. Mc. Graw-Hill., pp. 10-15, ISBN 978-607-315-0499-0499. México

[9] Vidal-Holguín, C. J. (2011). Introducción a la modelación matemática y optimización., Programación lineal, Programación No Lineal y Teoría de Redes: Formulación y solución de modelos, teoría de dualidad, análisis de sensibilidad, problema del transporte y problemas seleccionados de redes. Cali: Universidad del Valle, Escuela de Ingeniería Industrial y Estadística.

[10] Duran Guillermo (2006). Investigación de operaciones, modelos matemáticos y optimización. Seminario JUNAEB-DII. Enero de 2006. Universidad de Chile.

[11] Yois, S., y Pascuas, R. (2008). Modelamiento matemático aplicado al modelamiento espacial. Revista Sociedad de la Información. Art. N4. Bogotá, Colombia.

[12] González-Suárez, E., Quezada-Moreno, W., Peralta-Suárez, L. M., \& Concepción-Toledo, D. N. (2016). Modelación y optimización de procesos: impacto científico en la Industria Química y Fermentativa. Ecuador: Editorial UTC.

[13] Brito, M., Alemán, I., Fraga, E., Para, J. y Arias, R. (2011). Papel de la modelación matemática en la formación de ingenieros. Ingeniería Mecánica, 14(2), pp. 129-139.

[14] Rudd, D.F.; C.C. Watson (1968): Strategy of Process Engineering. Mc GrawHill. New York

[15] Torregrosa López, J. (2016). Conceptos básicos del modelado de procesos. Valencia: Universidad politécnica de Valencia. Departamento de Ingeniería Química y Nuclear.

[16] Plaza Gálvez, L.F. (2017). Modelación matemática en ingeniería. Revista de Investigación Educativa de la Rediech 7(13), pp. 47-57. Colombia 